\title{
Acceptance and usability of a home-based monitoring tool of health indicators in children of people with dementia: a Proof of Principle (POP) study
}

\author{
This article was published in the following Dove Press journal: \\ Patient Preference and Adherence \\ I August 2017 \\ Number of times this article has been viewed
}

\author{
April BCG Boessen' \\ Joan Vermeulen ${ }^{2}$ \\ Luc P de Witte ${ }^{3}$ \\ 'Research Centre for Technology \\ in Care, Faculty of Health, Zuyd \\ University of Applied Sciences, \\ Heerlen, the Netherlands; ${ }^{2}$ Lunet zorg \\ Eindhoven, the Netherlands; ${ }^{3}$ The \\ Innovation Centre, The University \\ of Sheffield, Sheffield, UK
}

Background: Large-scale cohort studies are needed to confirm the relation between dementia and its possible risk factors. The inclusion of people with dementia in research is a challenge, however, children of people with dementia are at risk and are highly motivated to participate in dementia research. For technologies to support home-based data collection during large-scale studies, participants should be able and willing to use technology for a longer period of time. Objective: This study investigated acceptance and usability of iVitality, a research platform for home-based monitoring of dementia health indicators, in 151 children of people with dementia and investigated which frequency of measurements is acceptable for them.

Methods: Participants were randomized to fortnightly or monthly measurements. At baseline and after 3 months, participants completed an online questionnaire regarding the acceptance (Technology Acceptance Model; 38 items) and usability (Post-Study System Usability Questionnaire; 24 items) of iVitality. Items were rated from 1 (I totally disagree) to 7 (I totally agree). Participants were also invited to take part in an online focus group (OFG) after 3 months of follow-up. Descriptive statistics and both two-sample/independent and paired $t$-tests were used to analyze the online questionnaires and a directed content analysis was used to analyze the OFGs.

Results: Children of people with dementia accept iVitality after long-term use and evaluate iVitality as a user-friendly, useful, and trusted technology, despite some suggestions for improvement. Overall, mean scores on acceptance and usability were higher than 5 (I somewhat agree), although the acceptance subscales "social influence" and "time" were rated somewhat lower. No significant differences in acceptance and usability were found between both protocol groups. Over time, "affect" significantly increased among participants measuring blood pressure fortnightly.

Conclusion: iVitality has the potential to be used in large-scale studies for home-based monitoring of health indicators related to the development of dementia.

Keywords: dementia, risk factors, e-health, telemonitoring, acceptance, usability

\section{Plain language summary}

To confirm the relation between dementia and possible risk factors, it is important to conduct studies among people with dementia with long follow-up periods. However, including people with dementia is difficult. Therefore, people at risk of developing dementia such as children of patients with dementia, could be included since they seem to be highly motivated to participate in research. Technologies can support the monitoring of possible risk factors of dementia. Before such technologies can be included in studies regarding the relation between dementia and its risk factors, it should be investigated whether participants are able and willing to use such
Correspondence: April BCG Boessen Research Centre for Technology in Care, Faculty of Health, Zuyd University of Applied Sciences, Henri Dunantstraat 2, 64I9PB Heerlen, the Netherlands

Tel +3I 880272120

Email april.boessen@zuyd.nl 
technologies for a long duration. This study shows that children of people with dementia accept the long-term use of iVitality, a monitoring tool measuring risk factors of dementia, based on online questionnaires and focus groups. The rate of acceptance did not differ between those measuring risk factors of dementia fortnightly or monthly. Therefore, we conclude that iVitality has the potential to be used for home-based monitoring of risk factors of dementia.

\section{Introduction}

The number of people who suffer from dementia is expected to increase rapidly in the coming years. ${ }^{1}$ Despite increased understanding of the causes of dementia, no cure or effective preventive interventions are available yet. Previous research suggests that interventions that aim to influence risk factors, such as uncontrolled blood pressure (BP), low mental and physical activity (AC), and obesity, could play a role in preventing dementia. ${ }^{2}$ Large-scale cohort studies with long-term follow-up are needed to confirm the relation between these risk factors and the onset of dementia. However, research which aims to study the preventive strategies that influence these risk factors needs to start before the onset of dementia and needs to include very large samples of older adults. This is a challenge in the field of research, including people with dementia. The inclusion of people with dementia in long-term follow-up studies is difficult due to deteriorating prognoses or even death. Furthermore, selecting a sample from the general population would require a large number of participants due to their relatively "low" risk of developing dementia. Therefore, we chose to recruit children of people with dementia, who have an increased risk of developing hypertension and dementia. ${ }^{3,4}$ Moreover, they are highly motivated to contribute to research about the prevention of dementia, because of their direct experiences with the impact of dementia. ${ }^{5,18}$

Technologies such as internet, smartphones, computers, sensors, and home-based monitoring devices can be used to support data collection during large-scale clinical studies. Such technologies are also increasingly used by middle-aged and older adults which provides opportunities to include them in research. ${ }^{6}$ If these technologies can facilitate participant recruitment and data collection, this can contribute to the development and study of evidence-based preventive strategies and treatment for the ageing population, including people with dementia.

iVitality is a research platform, consisting of a website, a smartphone-based application, and sensors which are connected to the smartphone. iVitality can be used for homebased long-term monitoring of several health indicators, ie, BP, AC, cognition (C), and lifestyle factors, that are associated with dementia, as shown in previous research., ${ }^{5-9}$ iVitality is intended to be used in the PROBE (PReservation Of Brian function in the Elderly) study, a large-scale trial on these health indicators and their relationship with dementia. In order to support such large-scale clinical studies regarding the etiology of dementia and potentially relevant prevention strategies, participants of such studies should be able and willing to use the platform for a longer period of time. Factors such as the usability of the platform, clearness of its interface, and its functional/technical adequacy might influence participants' willingness to use the platform. ${ }^{10}$ Furthermore, the frequency of health indicator measurements might influence participants' intention to use the platform. ${ }^{11}$ Therefore, the objectives of this Proof of Principle (POP) study are to gain insight into the long-term acceptance and usability of iVitality according to children of people with dementia, and to find out which frequency of measurements is acceptable for them.

\section{Methods}

\section{Design and participants}

The POP study had 6 months of follow-up. Potential participants were recruited via posters and flyers in memory clinics to reach children of people with dementia, who accompanied their parent to the memory clinic. Furthermore, potential participants were recruited via advertisements in the magazine and on the website of the Dutch Alzheimer Association. Participants were eligible for inclusion if they: 1) were children of people with late-onset dementia diagnosed as Alzheimer's disease, vascular dementia or mixed dementia, 2) were aged between 45 and 75 years old, 3) had no prior diagnosis of hypertension, and 4) were in possession of a smartphone with iOS or Android software (version 2.3.3. or higher). Children of people with dementia who wanted to take part in the POP study registered via the iVitality website. This website provided information about the study and after reading this information 195 participants registered online to participate. This study was approved by the Medical Ethical Committee of LUMC, the Netherlands (P11.131).

\section{Procedures and measurements}

A baseline (T1) assessment with a nurse practitioner or medical doctor from one of the participating memory clinics was scheduled with 151 participants who provided informed consent and were included in the study, based on the inclusion criteria. During this appointment, participants' office BP was measured and basic demographic characteristics, medication 
use, and medical history were recorded. Also, participants received an explanation about how to download the iVitality application on their own smartphone and how to use iVitality during the POP study. If necessary, they practiced this with the nurse practitioner. A BP measurement instrument was provided to all participants for the duration of the study. After participants downloaded the iVitality application they were randomly assigned to one of the two measurement protocols. Randomization was stratified for gender and performed in a 1:1 manner. Table 1 shows the measurement sequences of BP monitoring, $\mathrm{AC}$ monitoring, and $\mathrm{C}$ tests, and lifestyle questions (Q) of protocol 1 and protocol 2. For both protocols, $\mathrm{AC}, \mathrm{C}$, and lifestyle were measured on 4 consecutive days in the first and final week of the study. In addition, $\mathrm{C}$ and lifestyle were measured for 1 day each week in-between the first and final week. Differences between protocol groups concerned the frequency of BP measurement, which was measured monthly (on 2 consecutive days) for protocol 1 and fortnightly (on only 1 day) for protocol 2. A 1-day BP measurement consisted of two consecutive measurements in the morning and two in the evening. Via the iVitality application, participants received notifications with regard to these measurements. Participants used iVitality for 6 months. All data regarding $\mathrm{BP}, \mathrm{AC}, \mathrm{C}$, and $\mathrm{Q}$ were automatically uploaded to a central database which was password protected. If hypertension was diagnosed (average BP of 135/85 mmHg based on multiple measurements) the study doctor received an automated notification and informed the participant to visit his/her general practitioner (GP).

All participants received an online questionnaire at T1 and after 3 months of follow-up (T2) which contained

Table I Sequences of health indicator measurements per protocol group

\begin{tabular}{lll}
\hline Week number & Protocol I & Protocol 2 \\
\hline I (baseline) & 4d BP, 4d AC, 4d C+Q & 4d BP, 4d AC, 4d C+Q \\
3 & Id C+Q & Id BP, Id C+Q \\
5 & 2d BP, Id C+Q & Id BP, Id C+Q \\
7 & Id C+Q & Id BP, Id C+Q \\
9 & 2d BP, Id C+Q & Id BP, Id C+Q \\
II & Id C+Q & Id BP, Id C+Q \\
I3 & 2d BP, Id C+Q & Id BP, Id C+Q \\
15 & Id C+Q & Id BP, Id C+Q \\
17 & $2 d \mathrm{BP}$, Id C+Q & Id BP, Id C+Q \\
19 & Id C+Q & Id BP, Id C+Q \\
2 I & 2d BP, Id C+Q & Id BP, Id C+Q \\
23 & Id C+Q & Id BP, Id C+Q \\
25 & 4d BP, 4d AC, 4d C+Q & 4d BP, 4d AC, 4d C+Q \\
\hline
\end{tabular}

Abbreviations: d, days; BP, blood pressure; AC, physical activity; C, cognition; $Q$, lifestyle questions. questions regarding the acceptance and usability of iVitality. In addition, participants who participated between 3 and 6 months were invited to take part in an online focus groups (OFG) interview (T3) to gain insight into their experiences with the iVitality research platform.

\section{Online questionnaires}

An online questionnaire was used to measure the acceptance and usability of iVitality at T1 and T2. The questionnaire used to measure acceptance of iVitality was the Technology Acceptance Model (TAM) and its extensions developed by Venkatesch et al. ${ }^{12}$ The questionnaire consisted of 38 items divided over eight subscales: motivation (13 items), performance expectancy (five items), effort expectancy (four items), social influence (two items), affect (four items), trust (four items), self-efficacy (five items), and time (one item). According to the TAM, these concepts influence a person's intention to use a new technological innovation and by that the actual use in daily life. ${ }^{12}$ The complete acceptance questionnaire was included in the online questionnaire at T1 and T2. An adapted version of the Post-Study System Usability Questionnaire (PSSUQ) was used to measure usability of iVitality. ${ }^{10}$ This questionnaire consisted of 24 items divided over three subscales: system usefulness (nine items), information quality (eight items), and interface quality (eight items). These 24 items were included in the online questionnaire at $\mathrm{T} 1$ and T2. All items of the online questionnaires regarding acceptance and usability were rated on a scale from 1 (I totally disagree) to 7 (I totally agree). Higher scores indicated higher acceptance and usability. In addition, a "not applicable" answer category was added to all items.

\section{OFGs}

OFGs are feasible tools for collecting qualitative data. ${ }^{13,14}$ Two OFGs were conducted at T3 to collect user experiences with iVitality: one with participants, who were randomized to measurement protocol 1 (OFG 1), and one with participants, who were randomized to measurement protocol 2 (OFG 2). A web browser was used which could run on an MS Windows/ Web server platform. The OFGs took place in the last half of October 2014. All participants who had been using iVitality for at least 3 months at that moment were invited to take part in an OFG. Participants registered themselves and received a login and password from the moderator of the online platform (who was part of the research team) with which they could enter the OFG. Participants had access to the OFG platform for 2 weeks. During these 2 weeks, ten statements (one new statement on every weekday) regarding 
Table 2 Ten statements of the online focus groups

\begin{tabular}{|c|c|}
\hline \multicolumn{2}{|l|}{ Week I } \\
\hline Statement I & iVitality fits into my daily life \\
\hline Statement 2 & $\begin{array}{l}\text { I would like to use iVitality for } 2 \text { more years after } \\
\text { this study }\end{array}$ \\
\hline Statement 3 & $\begin{array}{l}\text { Contributing to scientific research is more important } \\
\text { than gathering information about my health }\end{array}$ \\
\hline Statement 4 & iVitality is a user-friendly system \\
\hline Statement 5 & iVitality influenced my health and lifestyle \\
\hline \multicolumn{2}{|l|}{ Week 2} \\
\hline Statement 6 & I am sufficiently able to use iVitality without help \\
\hline Statement 7 & $\begin{array}{l}\text { I would contact my general practitioner if my blood } \\
\text { pressure was too high }\end{array}$ \\
\hline Statement 8 & $\begin{array}{l}\text { I think that my privacy was guaranteed during the } \\
\text { use of iVitality }\end{array}$ \\
\hline Statement 9 & I trust iVitality to accurately present my health data \\
\hline Statement 10 & $\begin{array}{l}\text { What would you like to change about the way you } \\
\text { used iVitality during the study? }\end{array}$ \\
\hline
\end{tabular}

the use and experiences with iVitality were posted by the moderator (Table 2). Participants were invited by the moderator to respond to these statements and engage in an online discussion with each other. Participants could respond to all statements during the 2-week period at a time and place that was convenient for them. Consequently, communication between the participants was asynchronous. Participants were instructed not to mention any names for the sake of anonymity.

\section{Analyses \\ Online questionnaires}

Descriptive statistics were used to provide information on the $\mathrm{T} 1$ characteristics of the groups of participants assigned to measurement protocols 1 and 2. If participants filled out none of the items of the acceptance or usability questionnaire on $\mathrm{T} 1, \mathrm{~T} 2$ or filled out all items with "not applicable", they were excluded from the analyses. If participants filled out at least one question of both questionnaires, they were included in the analyses. In that case, missing items or "not applicable" answers on the questionnaire were imputed by the mean score of that item of all participants of the relevant measurement protocol at the particular time point. Cronbach's $\alpha$ was calculated for the subscales of the acceptance and usability questionnaire. Cronbach's $\alpha$ of the following subscales was below 0.7: motivation, social influence, affect, self-efficacy, and time. Deleting items for the subscales motivation and affect did not result in substantial improvements in the alphas and therefore no items were deleted. No items could be deleted for social influence and time, since both subscales consisted of only one or two items. Deleting items for the subscale selfefficacy did result in improvements in the alphas, however no items were deleted since mean scores of the subscale remained significantly unchanged after deleting items. Mean scores (SD) were calculated for subscales of the acceptance and usability questionnaires for both protocol groups separately. Independent samples $t$-tests were conducted to compare whether acceptance and usability was rated differently between both protocol groups. Paired samples $t$-tests were conducted to compare whether acceptance and usability was rated differently between measurement points ( $\mathrm{T} 1$ and $\mathrm{T} 2$ ). All analyses were performed using SPSS version 23.

\section{OFGs}

The moderator of the OFGs analyzed the data of the OFGs using a directed content analysis approach. Data were analyzed per statement for the two OFGs separately, to be able to detect differences in the experiences of participants who followed different measurement protocols.

\section{Results \\ Online questionnaires}

\section{Participant characteristics}

In total, 151 participants were included in the POP study and randomly assigned to two measurement protocols: 66 participants were randomized to measurement protocol 1 and 85 were randomized to protocol 2. Sixteen participants were excluded (four protocol 1 and twelve protocol 2), since they completed none of the questions of the TAM or PSSUQ at both measurement points. The resulting 135 participants were included in the analyses of the online questionnaires at T1 and T2 (62 protocol 1 and 73 protocol 2). T1 characteristics of these participants are provided in Table 3.

Missing items on the questionnaires of the included participants were imputed. For the acceptance questionnaire, $2.9 \%$ of the scores of all participants were imputed and $1.9 \%$ for the usability questionnaire. Overall, $2.5 \%$ of scores on the online questionnaires were imputed.

\section{Acceptance and usability questionnaires}

Table 4 shows the mean scores (SD) on the acceptance (motivation, performance expectancy, effort expectancy,

Table 3 Baseline characteristics of participants included in analyses of online questionnaires

\begin{tabular}{lll}
\hline & $\begin{array}{l}\text { Protocol I } \\
(\mathbf{n}=62)\end{array}$ & $\begin{array}{l}\text { Protocol 2 } \\
(\mathbf{n}=\mathbf{7 3})\end{array}$ \\
\hline Mean age in years (SD; min-max) & $56.4(4.9 ; 48-68)$ & $57.8(5.3 ; 49-72)$ \\
Gender (female/male) & $42 / 20$ & $52 / 21$ \\
Indication of hypertension & $16 / 46$ & $13 / 60$ \\
during study without prior & & \\
diagnosis (yes/no) & & \\
\hline
\end{tabular}


Table 4 Comparison of mean acceptance and usability scores between protocol groups at baseline (TI) and after 3 months of follow-up (T2)

\begin{tabular}{|c|c|c|c|c|}
\hline & \multirow{2}{*}{$\begin{array}{l}\text { Protocol I } \\
(n=62) \\
\text { Mean (SD) }\end{array}$} & \multirow{2}{*}{$\begin{array}{l}\text { Protocol } 2 \\
(n=73) \\
\text { Mean (SD) }\end{array}$} & \multicolumn{2}{|c|}{$\begin{array}{l}\text { Two-sample/ } \\
\text { independent } \\
t \text {-test }\end{array}$} \\
\hline & & & $t$ & $P$-value \\
\hline \multicolumn{5}{|l|}{ TI } \\
\hline Motivation & $5.09(0.45)$ & $4.97(0.52)$ & 1.44 & 0.15 \\
\hline Performance expectancy & $5.03(1.1)$ & $4.72(1.08)$ & 1.64 & 0.10 \\
\hline Effort expectancy & $5.74(1.01)$ & $5.64(0.94)$ & 0.59 & 0.56 \\
\hline Social influence & $3.05(1.23)$ & $2.89(1.10)$ & 0.79 & 0.43 \\
\hline Affect & $5.61(0.88)$ & $5.48(0.78)$ & 0.87 & 0.39 \\
\hline Trust & $5.95(0.78)$ & $5.95(0.82)$ & 0.00 & 0.80 \\
\hline Self-efficacy & $5.36(1.00)$ & $5.36(0.87)$ & -0.01 & 0.99 \\
\hline Time & $4.37(0.99)$ & $4.14(1.10)$ & 1.29 & 0.20 \\
\hline System usefulness & $6.53(0.78)$ & $6.60(0.54)$ & -0.66 & 0.51 \\
\hline Information quality & $6.37(0.74)$ & $6.47(0.63)$ & -0.90 & 0.37 \\
\hline Interface quality & $6.23(0.90)$ & $6.29(0.82)$ & -0.38 & 0.70 \\
\hline \multicolumn{5}{|l|}{ T2 } \\
\hline Motivation & $5.10(0.4 I)$ & $5.03(0.39)$ & 1.05 & 0.30 \\
\hline Performance expectancy & $5.05(0.98)$ & $4.70(1.07)$ & 1.94 & 0.06 \\
\hline Effort expectancy & $6.18(0.92)$ & $6.16(1.03)$ & 0.11 & 0.91 \\
\hline Social influence & $3.45(1.25)$ & 3.75 (1.29) & -1.36 & 0.18 \\
\hline Affect & $5.71(0.97)$ & $5.74(0.95)$ & -0.16 & 0.87 \\
\hline Trust & $6.04(0.83)$ & $6.07(0.79)$ & -0.19 & 0.85 \\
\hline Self-efficacy & $5.48(0.85)$ & $5.44(0.94)$ & 0.28 & 0.78 \\
\hline Time & $4.29(0.88)$ & $4.18(0.89)$ & 0.70 & 0.48 \\
\hline System usefulness & $6.51(0.63)$ & $6.59(0.52)$ & -0.84 & 0.40 \\
\hline Information quality & $6.23(1.00)$ & $6.35(0.70)$ & -0.86 & 0.39 \\
\hline Interface quality & $6.09(0.94)$ & $6.17(0.90)$ & -0.50 & 0.62 \\
\hline
\end{tabular}

social influence, affect, trust, self-efficacy, and time) and usability subscales (system usefulness, information quality, interface quality) at $\mathrm{T} 1$ and $\mathrm{T} 2$ for the two protocol groups separately. Table 4 also shows the $t$-scores and $P$-values of the independent samples $t$-tests which were conducted to compare the mean scores of both protocol groups at $\mathrm{T} 1$ and $\mathrm{T} 2$. The two-sample/independent $t$-tests revealed that there were no significant differences in acceptance and usability between both protocol groups. Overall, the mean scores on the acceptance and usability subscales were higher than 5 (I somewhat agree). The mean scores on the social influence and time subscale were somewhat lower for both protocol groups.

Mean scores of both protocol groups were also compared over time, eg, between measurement points $\mathrm{T} 1$ and $\mathrm{T} 2$. Table 5 shows the $t$-scores and $P$-values of the paired samples $t$-tests which were conducted. The paired $t$-tests revealed that scores on the effort expectancy subscale and social influence subscale significantly increased at T2 compared to T1 for both protocol groups. Mean scores on the affect subscale significantly increased between $\mathrm{T} 1$ and $\mathrm{T} 2$ for protocol group 2, meaning that only participants measuring BP fortnightly showed more affect toward using iVitality at T2.
Table 5 Comparison of mean acceptance and usability scores between baseline (TI) and after 3 months of follow-up (T2) per protocol group

\begin{tabular}{|c|c|c|c|c|}
\hline & \multirow{2}{*}{$\frac{\text { TI }}{\text { Mean (SD) }}$} & \multirow{2}{*}{$\frac{\mathrm{T2}}{\text { Mean (SD) }}$} & \multicolumn{2}{|c|}{ Paired $t$-test } \\
\hline & & & $t$ & $P$-value \\
\hline \multicolumn{5}{|l|}{ Protocol I $(n=62)$} \\
\hline Motivation & $5.09(0.45)$ & $5.10(0.4 \mathrm{I})$ & -0.13 & 0.90 \\
\hline Performance expectancy & $5.03(\mathrm{I} . \mathrm{I})$ & $5.05(0.98)$ & -0.14 & 0.89 \\
\hline Effort expectancy & $5.74(1.01)$ & $6.18(0.92)$ & -2.81 & $0.0 I^{*}$ \\
\hline Social influence & $3.05(1.23)$ & $3.45(1.25)$ & -3.05 & $0.00 *$ \\
\hline Affect & $5.61(0.88)$ & $5.7 \mid(0.97)$ & -0.88 & 0.38 \\
\hline Trust & $5.95(0.78)$ & $6.04(0.83)$ & -0.93 & 0.37 \\
\hline Self-efficacy & $5.36(1.00)$ & $5.48(0.85)$ & -1.10 & 0.28 \\
\hline Time & $4.37(0.99)$ & $4.29(0.88)$ & 0.57 & 0.57 \\
\hline System usefulness & $6.53(0.78)$ & $6.5 I(0.63)$ & 0.21 & 0.84 \\
\hline Information quality & $6.37(0.74)$ & $6.23(1.00)$ & 1.13 & 0.26 \\
\hline Interface quality & $6.23(0.90)$ & $6.09(0.94)$ & 1.36 & 0.18 \\
\hline \multicolumn{5}{|l|}{ Protocol $2(n=73)$} \\
\hline Motivation & $4.97(0.52)$ & $5.03(0.39)$ & -0.95 & 0.34 \\
\hline Performance expectancy & $4.72(1.08)$ & $4.70(1.07)$ & 0.20 & 0.84 \\
\hline Effort expectancy & $5.64(0.94)$ & $6.16(1.03)$ & -3.96 & $0.00^{*}$ \\
\hline Social influence & $2.89(1.10)$ & $3.75(1.29)$ & -5.82 & $0.00 *$ \\
\hline Affect & $5.48(0.78)$ & $5.74(0.95)$ & -2.46 & $0.02^{*}$ \\
\hline Trust & $5.95(0.82)$ & $6.07(0.79)$ & -1.19 & 0.24 \\
\hline Self-efficacy & $5.36(0.87)$ & $5.44(0.94)$ & -0.75 & 0.45 \\
\hline Time & $4.14(1.10)$ & $4.18(0.89)$ & -0.32 & 0.75 \\
\hline System usefulness & $6.60(0.54)$ & $6.59(0.52)$ & -0.02 & 0.99 \\
\hline Information quality & $6.47(0.63)$ & $6.35(0.70)$ & 1.51 & 0.14 \\
\hline Interface quality & $6.29(0.82)$ & $6.17(0.90)$ & 1.51 & 0.14 \\
\hline
\end{tabular}

Note: $* p<0.05$.

\section{OFGs}

\section{Participant characteristics}

In total, 32 participants registered for the OFGs and received a login and password. Eventually, 26 of them actively participated in an OFG: eleven in OFG 1 and 15 in OFG 2. Characteristics of these participants are provided in Table 6.

\section{Participant activity}

In OFG 1 participants posted 71 reactions in total during the 2-week period and the number of reactions per participant varied between two and eleven. In OFG 2 participants posted 118 reactions in total during the 2-week period and the number of reactions per participant varied between three and eleven. The information in Table 7 shows that eight

Table 6 Characteristics of online focus group (OFG) participants

\begin{tabular}{lll}
\hline & OFG I $(\mathbf{n}=\mathbf{I I})$ & OFG $2(\mathbf{n}=\mathbf{I 5})$ \\
\hline Mean age in years (SD; min-max) & $57.9(5.2 ; 5 \mathrm{I}-70)$ & $59.3(6.2 ; 52-70)$ \\
Gender (female/male) & $8 / 3$ & $10 / 5$ \\
Indication of hypertension & $0 / 1 \mathrm{I}$ & $4 / 1 \mathrm{I}$ \\
during study without prior & & \\
diagnosis (yes/no) & & \\
\hline
\end{tabular}


Table 7 Participants' activity on online focus group (OFG) forum

\begin{tabular}{lll}
\hline & $\begin{array}{l}\text { OFG I } \\
\text { (n=II) }\end{array}$ & $\begin{array}{l}\text { OFG 2 } \\
\text { (n=I5) }\end{array}$ \\
\hline Number of participants responding to all statements & 2 & 6 \\
Number of participants responding to 9 statements & I & 3 \\
Number of participants responding to 8 statements & 2 & 0 \\
Number of participants responding to 7 statements & 0 & 2 \\
Number of participants responding to 6 statements & 2 & 0 \\
Number of participants responding to 5 statements & I & 2 \\
Number of participants responding to 4 statements & I & I \\
Number of participants responding to 3 statements & I & I \\
Number of participants responding to 2 statements & I & 0 \\
\hline
\end{tabular}

participants (two in OFG 1 and six in OFG 2) responded to all statements whereas one participant (in OFG 1) responded only to two statements. Overall, the frequency of reactions on the forum decreased after week 1 in both groups. Reactions of participants in OFG 2 were more elaborate compared to reactions of participants of OFG 1 . In cases of participants replying to each other's reactions, instead of only voicing their opinion regarding the statement which was posted, they mostly agreed with each other.

\section{Participant experiences with iVitality}

Overall, participants in OFG 1 and OFG 2 agreed on most of the ten statements. Participants agreed that iVitality could be incorporated in their daily lives, although they preferred more flexible measurement moments and measurements outside their houses were difficult: "iVitality only fits use at home. I did not want to carry the BP device outdoors, which has led to some missing values" (protocol 1). Participants in both focus groups indicated that they had sufficient skills to use iVitality without help, although help being available was perceived as pleasant. Using iVitality encouraged participants to think about their health and lifestyle: "iVitality made me more aware of the importance of a healthy lifestyle, including being and staying active" (protocol 1). Gaining information on one's own health was equally or more important than contributing to scientific research for participants in OFG 1 , although participants in OFG 2 indicated the contribution to scientific research as more important (or equal to personal health information). All participants believed that their privacy was guaranteed by iVitality and that the results displayed were accurate. Furthermore, participants in both focus groups agreed that they would contact their GP if iVitality indicated a high BP and some actually did this: "Via the iVitality application, I received the notification that my BP was too high. So, I went to the doctor, but luckily no further action was required" (protocol 2). Participants in both focus groups had a somewhat negative view on the usability of iVitality. Participants indicated that the application sufficed for this study, but that improvements are needed in technical aspects (connection between BP measurement and smartphone, smartphone battery, restarting) and in the interface attractiveness. In OFG 1 half of the participants would have liked to continue their use of the iVitality application, while in OFG 2 none of the participants preferred this. Participants in OFG 2 would only continue their use of iVitality if feedback on the tests and games would be provided: "An added value would be to receive feedback regarding completed measurements. I would like to know in what way my own results compare to the standard" (protocol 2).

\section{Discussion}

This POP study evaluated the long-term acceptance and usability of iVitality according to children of people with dementia. It may be concluded that children of people with dementia accept iVitality after long-term use (6 months) and evaluated iVitality as a user-friendly, useful, and trusted technology, despite some technical and other suggestions for improvement. At T1 and T2, the level of acceptance and usability of iVitality did not differ between participants measuring health indicators monthly or fortnightly. When comparing acceptance and usability over time, participants conducting fortnightly health measurements showed a higher level of affection toward using iVitality at T2 compared to T1. The level of affection for iVitality of participants conducting monthly measurements did not change over time.

The results of this study are in line with the preliminary results of van Osch et al, ${ }^{5}$ who explored the usability of iVitality in four children of people with dementia, and showed the potential acceptance and usage of iVitality in larger user groups such as in this POP study. This finding is becoming more common in the light of the popularity of technology use and the increased uptake of innovative technologies by middle-aged adults. ${ }^{15}$ Middle-aged adults are getting used to technology and adopt and accept such technologies in health care settings more easily. This supports the potential of monitoring health indicators at home to prevent health problems, such as dementia. For example, some participants in this study contacted their GP when iVitality indicated a high BP and indicated that feedback on health data was very important. A suggestion for improvement was receiving feedback on the results displayed by iVitality and to be notified when further action is required. Such feedback is suggested to have the potential to influence patients' attitudes and health behavior as well. ${ }^{16}$ 
Attitude and behavior changes are of utmost importance for improving one's health. In this light, the type of motivation for the use of self-management tools plays a role in the actual outcomes of using such tools. Intrinsic motivation has been associated with positive health outcomes ${ }^{17}$ People's own choice, insight into personal health data, and contributing to research were important reasons to use iVitality. Subjective norms of important others did not seem to play a role in the decision to participate. This suggests that the intrinsic motivation to use iVitality was high among participants, while controlled motivation was low. Van Osch et $\mathrm{al}^{5}$ reported that the motivation to contribute to research might be a result of the unknown relation between dementia and hypertension, and indicated that addressing this relationship might stimulate self-monitoring. In addition to these motivations for self-monitoring, Wijsman et al ${ }^{18}$ monitored the adherence of participants of the POP study to the ascribed protocols. Overall, adherence to iVitality was acceptable $(>64 \%)$, although it was slightly better in participants measuring fortnightly $(71.4 \%)$ compared to participants performing monthly measurements (64.3\%). This rate of adherence is in agreement with previous research suggesting that motivation is the key to adherence with self-monitoring protocols. ${ }^{19-21}$ This study showed that participants measuring health indicators fortnightly showed a somewhat higher level of affection toward using iVitality over time. This is in line with the higher adherence rates among participants with fortnightly health indicator measurements reported by Wijsman et al. ${ }^{18}$ This finding should be put into perspective since all other acceptance and usability subscales did not differ between both measurement protocols, which indicates that acceptance and usability are fairly equal among participants measuring health indicators monthly and fortnightly. However, the slight preference for fortnightly health indicator measurements might be a result of participants developing a habit. Participants performing measurements more often (eg, fortnightly) may have been used to the procedure and may have experienced less burden due to the single-day measurements in comparison to the 2-day monthly measurements. This might especially apply to the elderly, who often have to cope with forgetfulness. A strength of this study was that the monitoring and feedback system was tested in the daily environment of the participants, which makes the results more realistic and provides more accurate and detailed information into the experiences and problems that can occur. With regard to the methodology, credibility and confirmability were increased by data triangulation. As participants were selected based on their presence in memory clinics at a certain time point, this may have introduced some selection bias. In addition, we did not measure information technology (IT) competences of participants, which might have influenced the acceptance and usability of iVitality. However, only participants in possession of a smartphone were included in the study. The findings also show that participants were highly motivated to participate, which may have influenced the results. Furthermore, response to the statements of the OFGs was disappointing and the asynchronous aspect of the OFGs led to little communication between participants, which is considered as one of the limitations of asynchronous OFGs compared to traditional focus groups (TFGs). Better instructions or fixed time periods of response might have contributed to an increase in participant activity and interaction. However, the asynchronous aspect and absence of time pressure is often valued for its convenience, since participants are unconstrained by time and place..$^{13,14}$ Moreover, OFGs provide benefits to the researchers, since lower recruitment costs and travel expenses are required and researchers save time due to automatic capture of data. Pitfalls of OFGs compared to TFGs are potential sampling bias due to computer-illiteracy or misinterpretation of information due to the lack of non-verbal signals..$^{13,14}$

The findings of this study in the light of previous research suggest that iVitality has the potential to be used in largescale clinical studies for home-based monitoring of health indicators related to the development of dementia, such as the PROBE study. The deployment of such a technology platform might contribute to the long-term monitoring of health indicators in children of people with dementia, the relation of these health indicators with dementia, and therefore the prevention of dementia. Furthermore, iVitality might be used for homebased monitoring among other patient groups for whom largescale studies with long follow-up periods are needed to show relations between health indicators and a disease. However, in order to realize the potential of iVitality in large-scale studies, a few issues should be addressed. Important suggestions for improvement were more flexible measurement moments and receiving feedback on the results displayed in the application. Furthermore, some technical shortcomings influenced the perceived usability of iVitality.

\section{Acknowledgments}

The work was supported by the Dutch Ministry of Health, Welfare and Sport and was enabled by ZonMw (project 627002001). In addition, the authors acknowledge the contributions of Simon Mooijaart and Edo Richard to the conception and design, the acquisition, analysis, and interpretation of the data. Furthermore, we would like to thank 
the participants for their time and motivation to participate in this study.

\section{Disclosure}

The authors report no conflicts of interest in this work.

\section{References}

1. World Health Organization [homepage on the Internet]. 10 facts on dementia. WHO; 2015 [updated April 2007]. Available from: http://www. who.int/features/factfiles/dementia/en/. Accessed June 26, 2017.

2. Barnes DE, Yaffe K. The projected effect of risk factor reduction on Alzheimer's disease prevalence. Lancet Neurol. 2011;10(9):819-828.

3. van Exel E, Eikelenboom P, Comijs H, et al. Vascular factors and markers of inflammation in offspring with a parental history of late-onset Alzheimer disease. Arch Gen Psychiatry. 2009;66(11): $1263-1270$

4. Green RC, Cupples LA, Go R, et al. Risk of dementia among white and African American relatives of patients with Alzheimer disease. JAMA. 2002;287(3):329-336.

5. van Osch M, Rovekamp A, Bergman-Agteres SN, et al. User preferences and usability of iVitality: optimizing an innovative online research platform for home-based health monitoring. Patient Prefer Adherence. 2015;9:857-867.

6. Denissen EG. Elderly and new technologies at home: allies or enemies? [master's thesis]. Twente: University of Twente; 2006.

7. Holm H, Nägga K, Nilsson ED, et al. Longitudinal and postural changes of blood pressure predict dementia: the Malmö Preventive Project. Eur J Epidemiol. 2017;32(4):327-336.

8. Blondell SJ, Hammersley-Mather R, Veerman JL. Does physical activity prevent cognitive decline and dementia? A systematic review and metaanalysis of longitudinal studies. BMC Public Health. 2014;14:510.

9. Cations M, Withall A, Low LF, Draper B. What is the role of modifiable environmental and lifestyle risk factors in young onset dementia? Eur J Epidemiol. 2016;31(2):107-124.

10. Lewis JR. Psychometric evaluation of the PSSUQ using data from five years of usability studies. International Journal of HumanComputer Interaction. 2002;14(3-4):463-488.
11. Slade EL, Williams M, Dwivedi Y. An extension of the UTAUT 2 in a healthcare context. Proceedings of the UK Academy for Information Systems Conference; March 19, 2013; UK.

12. Venkatesch V, Thong JYL, Xu X. Consumer acceptance and use of information technology: extending the unified theory of acceptance and use of technology. MIS Quarterly. 2012;36(1):157-178.

13. Tates K, Zwaanswijk M, Otten R, et al. Online focus groups as a tool to collect data in hard-to-include populations: examples from paediatric oncology. BMC Med Res Methodol. 2009;9:15.

14. Zwaanswijk M, van Dulmen S. Advantages of asynchronous online focus groups and face-to-face focus groups as perceived by child, adolescent and adult participants: a survey study. BMC Res Notes. 2014;7:756.

15. Statistics Netherlands (Centraal Bureau voor de Statistiek) [homepage on the Internet]. Internet; access, use and facilities. CBS; 2016. Available from: http://statline.cbs.n1/Statweb/publication/?VW=T\&DM=SLNL\& $\mathrm{PA}=83429 \mathrm{NED} \& \mathrm{D} 1=20-24 \& \mathrm{D} 2=3-6 \& \mathrm{D} 3=0 \& \mathrm{D} 4=0,1 \& \mathrm{HD}=160622-$ 0920\&HDR=G3,T,G2\&STB=G1. Accessed June 26, 2017.

16. DiClemente CC, Marinilli AS, Singh M, Bellino LE. The role of feedback in the process of health behavior change. Am J Health Behav. 2001;25(3):217-227.

17. Levesque CS, Williams GC, Elliot D, Pickering MA, Bodenhamer B, Finley PJ. Validating the theoretical structure of the Treatment Self-Regulation Questionnaire (TSRQ) across three different health behaviors. Health Educ Res. 2007;22(5):691-702.

18. Wijsman LW, Richard E, Cachucho R, de Craen AJ, Jongstra S, Mooijaart SP. Evaluation of the use of home blood pressure measurement using mobile phone-assisted technology: the iVitality proof-ofprinciple study. JMIR Mhealth Uhealth. 2016;4(2):e67.

19. Chaudhry SI, Mattera JA, Curtis JP, et al. Telemonitoring in patients with heart failure. N Engl J Med. 2010;363(24):2301-2309.

20. Kerby TJ, Asche SE, Maciosek MV, O'Connor PJ, Sperl-Hillen JM, Margolis KL. Adherence to blood pressure telemonitoring in a clusterrandomized clinical trial. J Clin Hypertens (Greenwich). 2012;14(10): 668-674.

21. Marshall A, Medvedev O, Antonov A. Use of a smartphone for improved self-management of pulmonary rehabilitation. Int J Telemed Appl. 2008:753064.
Patient Preference and Adherence

\section{Publish your work in this journal}

Patient Preference and Adherence is an international, peer-reviewed, open access journal that focuses on the growing importance of patient preference and adherence throughout the therapeutic continuum. Patient satisfaction, acceptability, quality of life, compliance, persistence and their role in developing new therapeutic modalities and compounds to optimize

\section{Dovepress}

clinical outcomes for existing disease states are major areas of interest for the journal. This journal has been accepted for indexing on PubMed Central. The manuscript management system is completely online and includes a very quick and fair peer-review system, which is all easy to use. Visit http://www. dovepress.com/testimonials.php to read real quotes from published authors. 